\title{
Microstructure evolution and liquid phase separation in Ta-O hypermonotectic melts during laser-cladding
}

\author{
*Hai-ou Yang, Meng Wang, Xin Lin, and Wei-dong Huang \\ State Key Laboratory of Solidification Processing, Northwestern Polytechnical University, Xi'an, Shaanxi 710072, PR China \\ MIIT Key Laboratory of Metal High Performance Additive Manufacturing and Innovative Design, Northwestern Polytechnical \\ University, Xi'an, Shaanxi 710072, PR China
}

\begin{abstract}
A three-layer $\mathrm{Ta}_{2} \mathrm{O}_{5}$-containing coating was successfully fabricated by laser cladding on a pure Ta substrate. The maximum thickness of such a coating is about $1.6 \mathrm{~mm}$. The microstructure, phase constitution and elemental distribution in the coating were investigated. Results show that the coating has been metallurgically bonded to the Ta substrate and the microstructure exhibits a graded change along the deposition direction from Ta substrate to the top of coating. In the layers I and II of the graded coating, the microstructure evolution can be confirmed as a result of hypomonotectic reaction, but in the layer III it was formed by hypermonotectic reaction. At the top of coating, the microstructure was still homogeneous although liquid phase separation had occurred, which can be attributed to the fact that the O-rich droplets do not have enough time to float at high cooling rate. The theoretical calculation results show that during laser cladding, the solidification time of the melt pool was less than $0.1 \mathrm{~s}$, which fits well with the results from the experiment.
\end{abstract}

Key words: Laser cladding; liquid phase separation; microstructure evolution; homogeneous structure
CLC numbers: TG174.44
Document code: A
Article ID: 1672-6421 2018 03-222-06

\begin{abstract}
I: n recent years, $\mathrm{Ta}_{2} \mathrm{O}_{5}$ ceramic coating has been used for a variety of engineering applications ${ }^{[1-2]}$ because it exhibits many attractive properties, such as high dielectric constant, melting point of $1,872{ }^{\circ} \mathrm{C}$, high transmittance, low optical loss and self-emission at high temperatures ${ }^{[3,4]}$. At the same time, $\mathrm{Ta}_{2} \mathrm{O}_{5}$ has received increasing attention for use as an excellent biomaterial owing to its good corrosion and wear resistance, as well as excellent biocompatibility ${ }^{[5]}$. Besides, it is also a very important reason that the formation of highly stable $\mathrm{Ta}_{2} \mathrm{O}_{5}$ oxide can greatly reduce the concentration of metal release from the surface oxide layer ${ }^{[6]}$. Thus the corrosion resistance of the passive film is highly dependent on the thickness of the layer formed ${ }^{[7]}$.

Many surface deposition techniques, such as thermal oxidation ${ }^{[8]}$, physical vapour deposition ${ }^{[9]}$, plasma
\end{abstract}

\footnotetext{
*Hai-ou Yang
}

Male, born in 1976, assistant professor. His research interests mainly focus on the laser additive manufacturing

Corresponding author: Wei-dong Huang

E-mail: huang@nwpu.edu.cn

Received: 2018-04-06; Accepted: 2018-05-03 spraying ${ }^{[10]}$, micro arc oxidation ${ }^{[11]}$, laser surface modification including laser re-melting ${ }^{[12-14]}$, laser alloying ${ }^{[12]}$ and laser cladding ${ }^{[15]}$ have been used to fabricate hard and wear resistant coatings. Among these, thermal oxidation, physical vapour deposition and micro arc oxidation have the disadvantage of only forming relatively thin protective coatings ${ }^{[8-9,11]}$. Although thermal spraying can produce a thick coating, it has a major drawback of poor substrate-coating bond strength. As for laser surface modification, it has the advantages of forming a strong substrate-coating interface, coating designed areas of a component, and readily changing the composition of the coating during laser cladding. Indeed, laser surface modification can be used to prepare bulk or thick $\mathrm{Ta}_{2} \mathrm{O}_{5}$ coating.

In this paper, thick $\mathrm{Ta}_{2} \mathrm{O}_{5}$-containing coating on $\mathrm{Ta}$ substrate was successfully fabricated by laser cladding, where a functional gradient structure could be obtained by controlling the contents of $\mathrm{Ta}, \mathrm{Ta}_{2} \mathrm{O}_{5}$ powder. The microstructure of the coating was observed and the behaviour of liquid-phase separation was described according to the theoretical calculation. 


\section{Experimental}

\subsection{Laser-clad coating preparation}

Raw materials for laser cladding were $99.9 \mathrm{wt} . \%$ commercial pure $\mathrm{Ta}(\mathrm{CP} \mathrm{Ta})$ and $\mathrm{Ta}_{2} \mathrm{O}_{5}$ powder with average diameter less than $45 \mu \mathrm{m}$. Firstly, pure Ta powder was preplaced on a commercial tantalum $(99.9 \%)$ substrate to form a powder bed with a thickness of about $1.0 \mathrm{~mm}$. A $3-\mathrm{kW}$ continuous-wave fiber laser (IPG 3000CL) was used to melt this powder bed, and then the first layer of coating with a thickness of about 0.3-0.4 mm was formed on the surface of CP-Ta substrate. Subsequently, a mixed $\mathrm{Ta}+\mathrm{Ta}_{2} \mathrm{O}_{5}$ powder bed with a volume percentage of 1:1 was preplaced on the first layer, and the same laser-cladding treatment was used. Finally, laser cladding was carried out on a pure $\mathrm{Ta}_{2} \mathrm{O}_{5}$ powder bed with a thickness of about $2 \mathrm{~mm}$. Considering that a $\mathrm{Ta}_{2} \mathrm{O}_{5}$-containing coating would be obtained, all the laser-cladding experiments were performed in air. During laser-cladding, the laser power was kept constant at 1,800 W. The laser beam size was fixed at $3 \mathrm{~mm}$. The laser scanning speed was $5 \mathrm{~mm} \cdot \mathrm{s}^{-1}$, while a $50 \%$ overlap condition for multi-track was employed.

\subsection{Coating characterization}

The specimens for microscopic study were ground with a

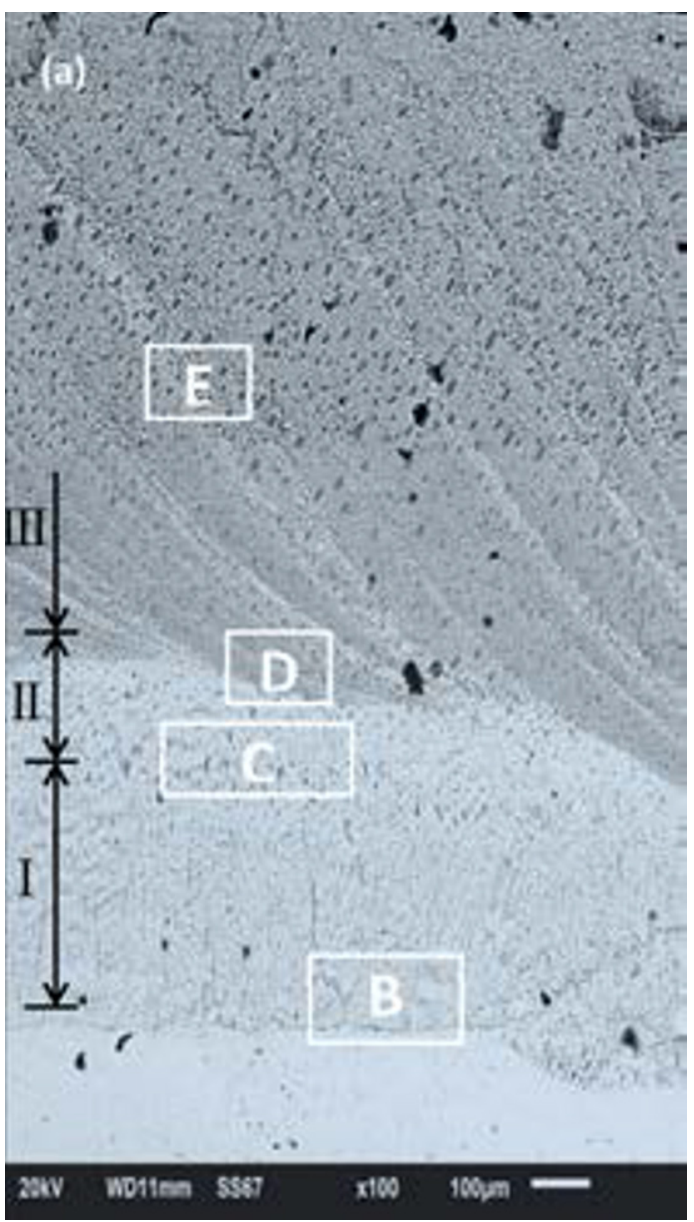

series of emery papers and finally polished with $0.25 \mu \mathrm{m}$ diamond abrasives. The phase and crystal structures of the laser-cladded coating were analyzed using the X-ray diffraction (XRD) technique with a XRD-7000 diffractometer (Shimadzu Ltd., Japan) using $\mathrm{Cu}-\mathrm{K} \alpha$ radiation at $40 \mathrm{kV}$ and $30 \mathrm{~mA}$. The microstructure of the coating was studied using a JEOL JSM6510A (JEOL Ltd., Japan) scanning electron microscope equipped with energy dispersive x-ray spectroscopy (EDS). In this paper, all the backscattered electron images of the specimen were in the unetched condition.

\section{Results and discussion}

\subsection{Microstructural observation}

Figure 1 shows the whole laser-clad coating on a Ta substrate (Fig.1a), the composition gradient across the coating (Fig.1b) and Ta-O phase diagram (Fig.1c). Figure 1(a) indicates the longitudinal section macrograph of a whole coating fabricated on the surface of CP tantalum substrate by laser-cladding. The maximum thickness of such a coating is about $1.6 \mathrm{~mm}$, which contains a small number of pores. The bonding zone shows that such a $\mathrm{Ta}_{2} \mathrm{O}_{5}$-containing coating has a good metallurgical
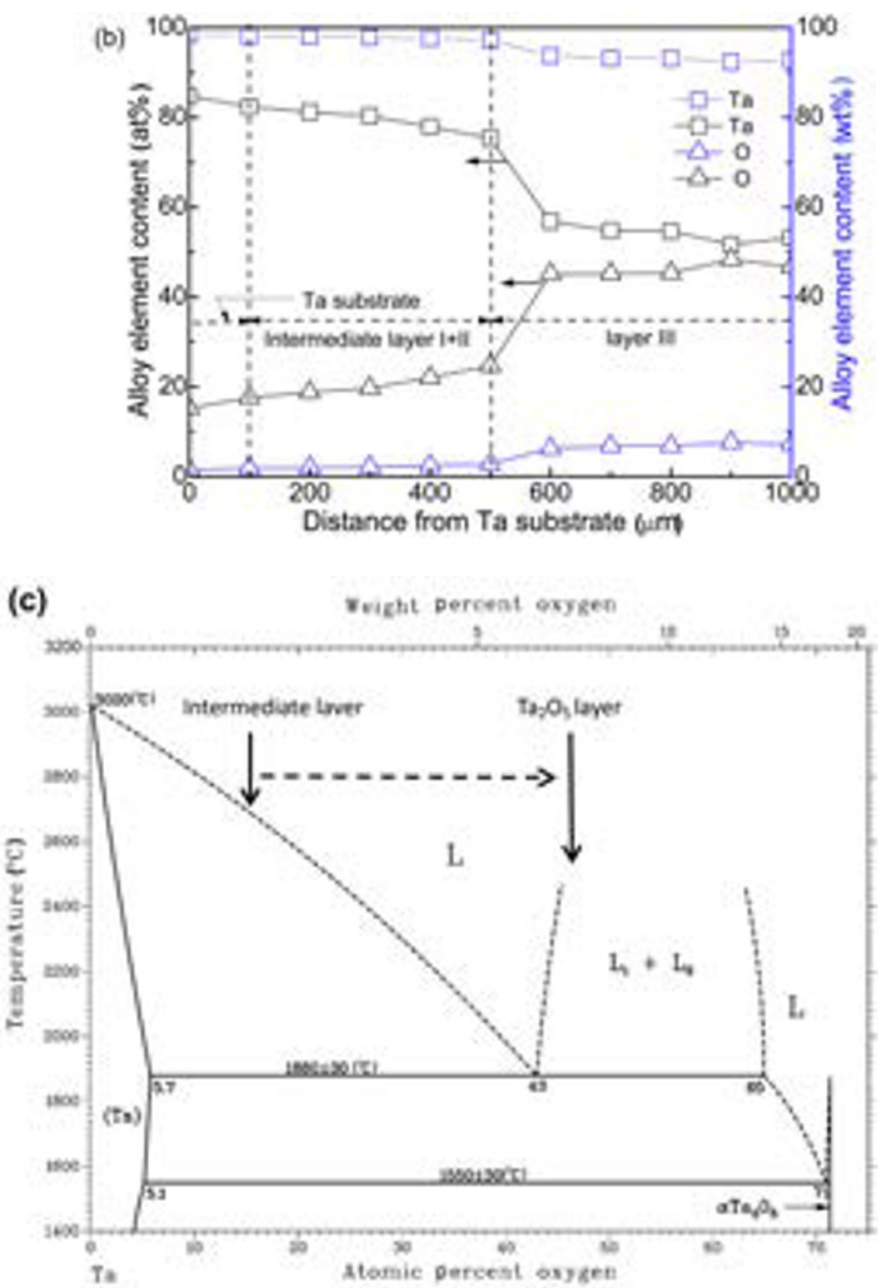

Fig. 1: Cross section laser-cladded coating on a Ta substrate (a), element distribution along the distance from Ta substrate to the top of clad coating (b), and the corresponding composition of different layers in the coating inserted in Ta-O binary phase diagram (c) 
bond with the tantalum substrate. The coating can be regarded as the combination of 3 layers according to colour contrast and microstructure on the image, which is in accordance with the experimental process of laser cladding. The results of the EDS analysis (Fig.1b) along the vertical direction of the coating shows that with the deposition of pure Ta, mixed $\mathrm{Ta}+\mathrm{Ta}_{2} \mathrm{O}_{5}$ and pure $\mathrm{Ta}_{2} \mathrm{O}_{5}$, the concentration of oxygen reached 48.33 at.\% towards the top surface of the coating. Within such a compositional range, and according to the Ta-O phase diagram (Fig.1c), a series of reactions, hypomonotectic, monotectic and hypermonotectic, should have occurred.

Figure 2 presents the XRD patterns of the laser-clad coating and tantalum substrate. Different from tantalum substrate with solely Ta solid solution, the coating shows a mixture of tantalum solid solution and $\mathrm{Ta}_{2} \mathrm{O}_{5}$ phase. The peaks of tantalum solid solution shift to low angles, indicating the lattice distortion of tantalum solid solution in the coating occurred. Moreover, the XRD result shows no evidence of the presence of other type of tantalum oxide in the coating, revealing that a $\mathrm{Ta}_{2} \mathrm{O}_{5}$-containing coating can be successfully fabricated on the tantalum substrate by laser cladding.

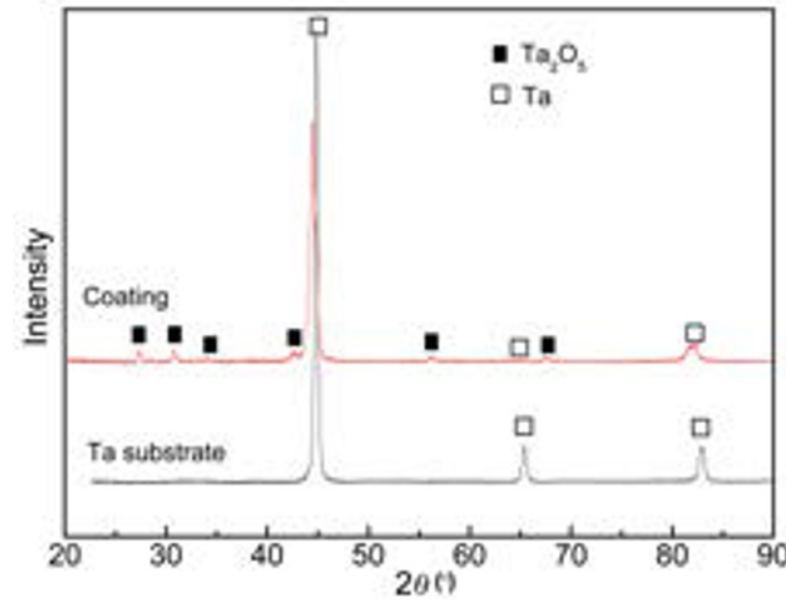

Fig. 2: XRD patterns obtained from Ta substrate and laser-clad coating

\subsection{Phase evolution}

To investigate the phase evolution during laser deposition, the microstructure along the compositional gradient was studied using SEM. Figure 3(a-e) corresponding to the regions labelled B-E in Fig.1(a) show the backscattered SEM images of the coating along the direction perpendicular to the substrate.

In layer I, the microstructure exhibits a columnar growth character, and there are a large number of dark particles surrounded by the light colour phases (Fig.3a). EDS surface analysis shows that in layer I, the oxygen content is up to 18.81 at. $\%$, illustrating severe high temperature oxidation has occurred due to laser-cladding of pure Ta powder in air. Moreover, EDS analysis also shows the oxygen content in the bright phase is as high as 6.91at.\%. Ta in the dark particle reaches 33at.\% which is approximately close to stoichiometry of $\mathrm{Ta}_{2} \mathrm{O}_{5}$. Combining the XRD, EDS analysis with the backscatter electron images, the light colour phase can be identified as Tarich solid solution, the dark phase is $\mathrm{Ta}_{2} \mathrm{O}_{5}$. According to Ta-O binary phase diagram ${ }^{[16]}$, in the composition position labelled with the arrow (Fig.1c), as the temperature drops, Ta-rich solid solution $\mathrm{Ta}(\mathrm{O})$ solidifies firstly as the primary phase from Ta-O melt, and grows in a columnar form. At the same time, oxygen is rejected into the residual liquid. As for high Ta, $\mathrm{O}$ content in different phases, these can be attributed to solute trapping during laser rapid forming. The higher oxygen content should be responsible for the diffraction peaks of tantalum solid solution in the coating shifting to low angles.

With the increase of the distance away from tantalum substrate, the microstructure between the layers I and II does not change significantly (Fig. 3b), and the bonding interface between layers I and II is also good. EDS surface analysis shows that the oxygen content in layer II is up to 24.63at.\%, higher than that in layer I. EDS analysis also shows the oxygen content in the Ta layer is as high as 7.95at.\%, and Ta in the $\mathrm{Ta}_{2} \mathrm{O}_{5}$ layer reaches 30.43 at.\%. Although the oxygen content is higher in this layer formed by laser-cladding of the mixed $\mathrm{Ta}+$ $\mathrm{Ta}_{2} \mathrm{O}_{5}$ powder, the solidification behaviour seems to be similar to that in the first layer except the size of $\mathrm{Ta}_{2} \mathrm{O}_{5}$ particles becomes larger. The fact that the size of $\mathrm{Ta}_{2} \mathrm{O}_{5}$ particles becomes larger can be understood as follows. As Ta-rich solid solution solidified firstly as primary phase, the oxygen atoms were rejected into residual liquid, which would cause local oxygen content in the residual liquid to increase. Therefore, the phase separation and aggregation of O-rich droplets was inevitable, and then the dark phase with a bigger size formed in a short solidification time.

With a further increase of the distance, the microstructure and colour contrast in layer III (the top of coating) is fully different from those in layers I and II (Fig. 1a). In layer III, the trace of molten pool movement during laser cladding can be seen clearly (Fig. 1a). The bonding interface between layers II and III is very good (Fig. 3c), and the oxygen content increases significantly (Fig.1b). In layer III, a number of dark ellipsoidal spheres with different sizes are found to be embedded in the matrix, and can be confirmed as $\mathrm{Ta}_{2} \mathrm{O}_{5}$ by EDS. In layer III, the tantalum content varies slightly from 51.67 at. $\%$ to 54.85 at. $\%$, higher than that in $\mathrm{Ta}_{2} \mathrm{O}_{5}$. The high Ta content in layer III should be the result of laser remelting of layer II and the Ta has mixed with melted $\mathrm{Ta}_{2} \mathrm{O}_{5}$ to form a liquid solution. The morphology of $\mathrm{Ta}_{2} \mathrm{O}_{5}$ phase is ellipsoidal, and their size is not the same (Fig. 3d). It is believed that $\mathrm{Ta}_{2} \mathrm{O}_{5}$ phase particles with different sizes are the products of O-rich droplets according to the solidification sequence of the Ta-O hypermonotectic melt. In other words, such a result reveals that the liquid phase separation should occur in the Ta-O hypermonotectic melt in layer III. However, during laser cladding, it needs to be further discussed whether the nucleation, growth and settlement of O-rich droplets in the Ta-O hypermonotectic melt can occur under the condition of rapid solidification. 

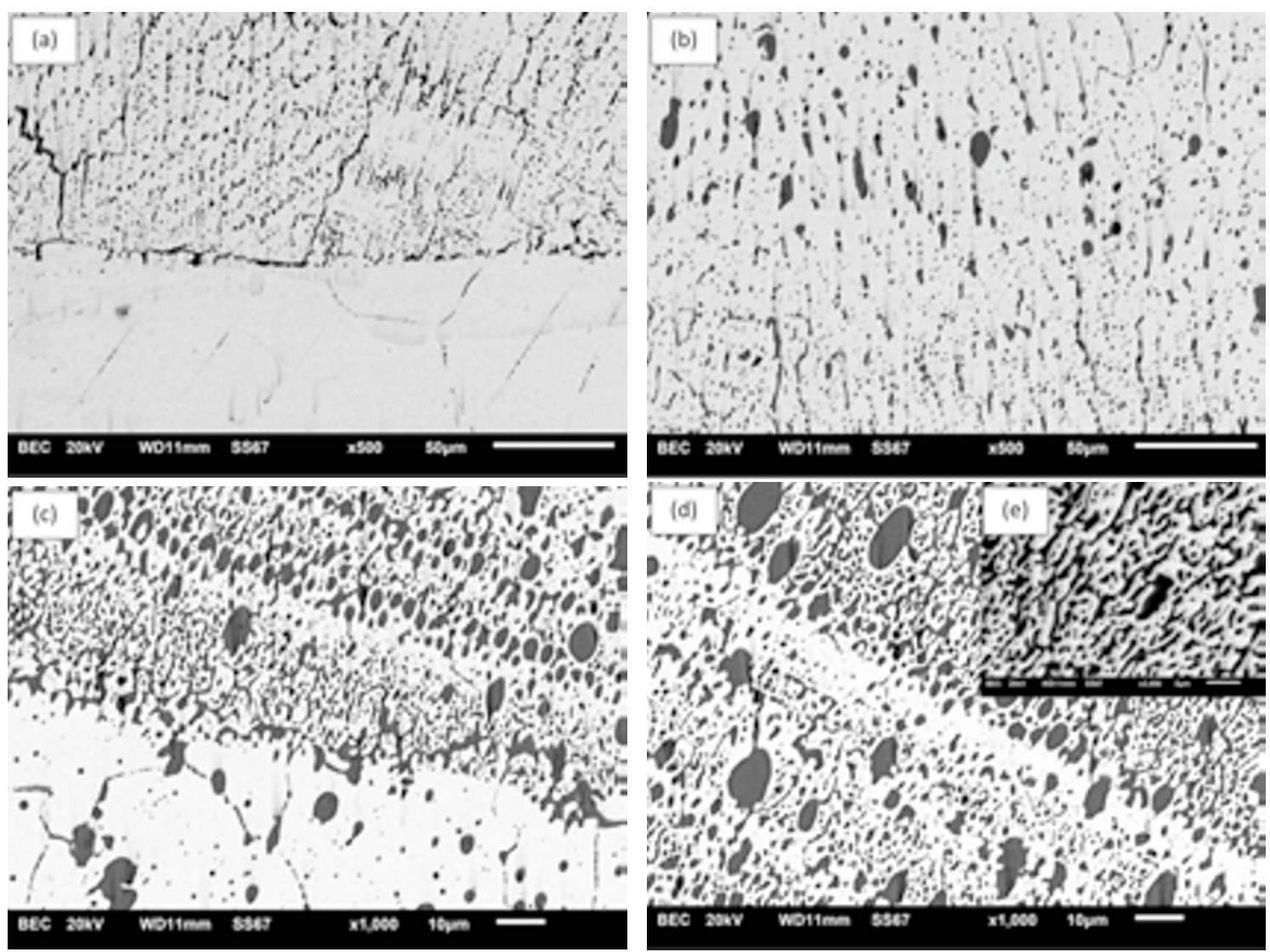

Fig. 3: Bonding interface of substrate/layer I (a), I/II (b) and II/III (c); the image showing the microstructure of Ta-O hypermonotectic alloy in layer III (d) and a higher magnification (e) of microstructure by monotectic reaction inserted in (d)

\section{3 liquid-phase separation}

According to the Ta-O phase diagram (Fig. 1c), as the temperature drops, the Ta-O hypermonotectic melt passes through the immiscibility gap and liquid-liquid phase separation occurs, i.e. $\mathrm{L} \rightarrow \mathrm{L}_{1}\left((\right.$ Ta-rich $)+\mathrm{L}_{2}(\mathrm{O}$-rich $)$. The Ta-rich $\mathrm{L}_{1}$ droplets will sink towards the bottom of the melt pool because of its higher density. As the melt temperature drops further, a monotectic reaction, $\left[\mathrm{L}_{1}(\right.$ Ta-rich $) \rightarrow \mathrm{Ta}(\mathrm{O})+\mathrm{L}_{2}(\mathrm{O}$-rich $\left.)\right]$ takes place, and oxygen will be rejected into residual liquid. Finally, this residual liquid solidifies, and then $\rightarrow \alpha-\mathrm{Ta}_{2} \mathrm{O}_{5}$ forms. Once O-rich droplets nucleate and grow, they will float upward due to lower density.

In fact, the three stages of liquid-liquid phase separation in the Ta-O hypermonotectic melt, namely nucleation, growth and floating, are not factually isolated from each other. It is very important for the study on solidification behaviour to obtain a mathematic relation among three stages in the immiscible hypermonotectic melt. L. Ratke et al. ${ }^{[17]}$ described the whole process of liquid-liquid separation by using a method of statistics under equilibrium solidification condition. The whole process of liquid-phase separation in Ta-O hypermonotectic melt can be described according to the following equation:

$$
\frac{\partial f(r, t)}{\partial t}-u(r) \frac{\partial f(r, t)}{\partial x}+\frac{\partial}{\partial r}\left(\frac{d r}{d t} \cdot f(r, t)\right)=Q(r, x, t)
$$

where $f(r, t)$ is defined as a droplet radius distribution function, $f(r, t) d r$ is the number of droplets per unit volume within a size range of $(r, r+d r)$, and $Q(r, x, t)=I_{s s}(t) \cdot b(r) \cdot q(x)$ in which $Q(r, x, t)$ is a source function, $I_{s s}(t)$ is the steady state nucleation rate, $b(r)$ is the droplets nucleate with a certain size spectrum, $q(x)$ is a function that the nucleation might depend on position in a sample. A mathematical solution of Eq. 1 under a condition of steady-state nucleation was achieved by F. John ${ }^{[18]}$, and then the volume fraction of the second phase droplets can be written as follows:

$$
\Phi(t)=\frac{4 \pi}{3} \int_{0}^{r_{\max }} r^{3} f(r, t) d r=\frac{4 \pi I_{S S}^{0}}{15 D S}(2 D S t)^{5 / 2}
$$

where the coefficient of diffusion $D$ and the supersaturation $S$ are the functions of melt temperature. The volume fraction of the droplets in the melt is closely related to both the solidification time and temperature.

In the present work, the magnitude of cooling rate is relative to the length of the solidification time in which the nucleation, growth and floating of O-rich droplets will take place adequately. Therefore, it can be considered that the volume fraction of O-rich droplets can be controlled by the cooling rate. Although the liquid-phase separation can not be fully inhibited, a solidification structure at the top of the coating can still be considered homogeneous under the condition of laser cladding (Fig.1a). If the settlement volume fraction of O-rich droplets is controlled within a certain range, for example less than 10vol.\%, the change of $\Phi(t)$ in Eq. 2 with the solidification time $t$ will be rewritten as: 


$$
(D S)^{\frac{3}{2}} \cdot t^{\frac{5}{2}}=1
$$

In the present work, the supersaturation $S$ is defined as:

$$
\mathrm{S}=\frac{C_{0}-C_{L 1}}{C_{L 1}-C_{L 2}}
$$

where $C_{L 1}$ and $C_{L 2}$ are the compositions of Ta-rich and O-rich droplets at the temperature $T$ in the immiscible gap.

Figure 4 shows the relationship between the supersaturation $\mathrm{S}$ and the temperature $T$ by calculating according to KGT model ${ }^{[19]}$. The thermal physical parameters required for calculation are shown in Table 1.

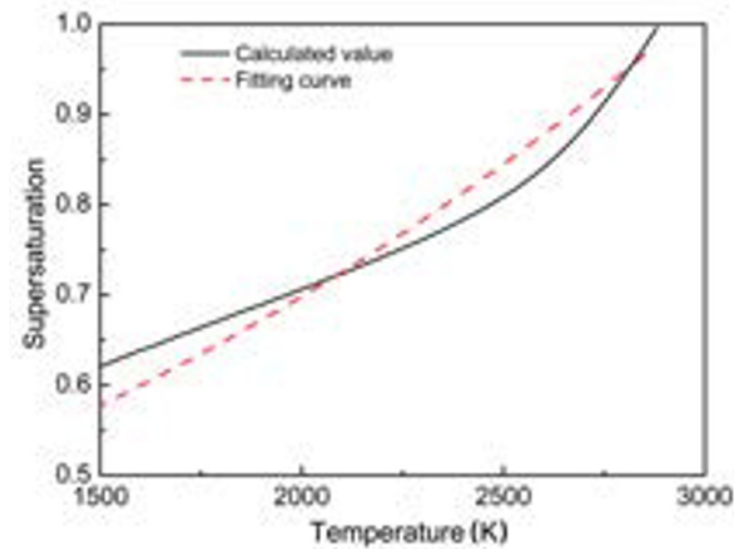

Fig. 4: Solute supersaturation vs. temperature of Ta-O hypermonotectic melt

Table 1: Thermodynamical parameters of Ta-O hypermonotectic alloy

\section{Thermodynamical parameters}

Ta content in melt, $x_{\mathrm{Ta}}($ at. $\%)$

Oxygen content in melt, $x_{\mathrm{O}}($ at. $\%)$ 55.6

Molar volume of Ta atom, $\Omega_{\mathrm{Ta}}\left(\mathrm{m}^{3} \cdot \mathrm{mol}^{-1}\right)$

Molar volume of $\mathrm{O}$ atom, $\Omega_{\mathrm{O}}\left(\mathrm{m}^{3} \cdot \mathrm{mol}^{-1}\right)$ $18.7 \times 10^{-9}$

Diffusion coefficient of oxygen in tantalum, $D$

Atomic transition distance, $\lambda(\mathrm{m})$

$3.5 \times 10^{-5}$

Tantalum density, $\rho_{\text {Ta }}\left(\mathrm{kg} \cdot \mathrm{m}^{-3}\right)$

$16.654 \times 10^{3}$

Oxygen density, $\rho_{\circ}\left(\mathrm{kg} \cdot \mathrm{m}^{-3}\right)$

1.429

Mole mass of tantalum, $M_{\mathrm{Ta}}$

180.9

Mole mass of oxygen, $M_{\mathrm{O}}$

Binodal decomposition temperature

$T_{\text {bin }}(\mathrm{K})$
The relationship between the saturation of the melt with the temperature by fitting can be expressed as:

$$
S=0.01245+0.3157 \exp \left(\frac{T}{2579.13}\right)
$$

By replacing Eq. 5 into Eq. 3, the change of the temperature with the cooling time can be obtained, and presents in Fig. 5. It is found that during laser cladding, the solidification time of the melt pool is less than $0.1 \mathrm{~s}$, and the cooling rate is located at a range of about $(4.73-6.56) \times 10^{5} \mathrm{~K} \cdot \mathrm{s}^{-1}$, which is very close to the values used for predicting the laser surface melting process ${ }^{[21,}$ ${ }^{22]}$. At such a cooling rate, although the liquid phase separation is inevitable, the O-rich droplets cannot float fully in such a short time. As a result, the ellipsoidal $\mathrm{Ta}_{2} \mathrm{O}_{5}$ phases finally disperse in the $\mathrm{Ta}(\mathrm{O})$ matrix, and a homogeneous solidification of $\mathrm{Ta}-\mathrm{O}$ melt can be obtained during laser cladding.

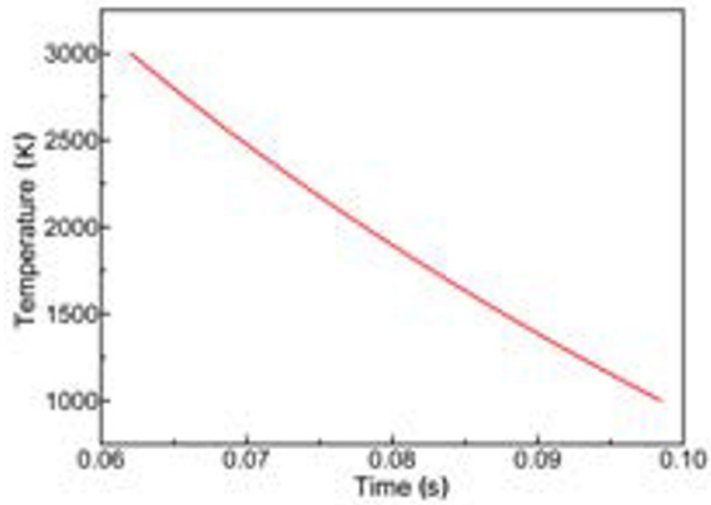

Fig. 5: Relationship between temperature with time obtained by theoretical calculation

\section{Conclusions}

(1) A graded $\mathrm{Ta}_{2} \mathrm{O}_{5}$-containing coating with three layers can be successfully fabricated using the preplaced powder bed method on CP tantalum substrate by laser cladding. The bonding interface between substrate and coating is considered to be good.

(2) In layers I and II of the graded coating, the microstructure evolution can be confirmed as a result of hypomonotectic reaction, but in layer III it is formed by hypermonotectic reaction.

(3) Although liquid phase separation has occurred, the homogeneous structure can be still obtained at the top of the coating, which can be attributed to the fact that the O-rich droplets do not have enough time to float at the high cooling rate. Theoretical calculation results (during laser cladding, the solidification time of melt pool is less than $0.1 \mathrm{~s}$ ) are consistent with the results observed in the experiment.

\section{References}

[1] Chaneliere C, Autran J L, Devine R A B. Tantalum pentoxide 
$\left(\mathrm{Ta}_{2} \mathrm{O}_{5}\right)$ thin films for advanced dielectric applications. Marer. Sci. Eng. R., 1998, 22: 269-322.

[2] Li Y, Zhao T T, Wei S B, et al. Effect of $\mathrm{Ta}_{2} \mathrm{O}_{5} / \mathrm{TiO}_{2}$ thin film on mechanical properties. corrosion and cell behavior of the NiTi alloy implanted with tantalum. Mater. Sci. Eng. C, 2010, 30: 1227-1235.

[3] Shang $P$, Xiong $S M$, Li L H, et al. Investigation on thermal stability of $\mathrm{Ta}_{2} \mathrm{O}_{5}, \mathrm{TiO}_{2}$ and $\mathrm{Al}_{2} \mathrm{O}_{3}$ coatings for application at high temperature. Appl. Surf. Sci., 2013, 285: 713-720.

[4] Franco F D, Santamaria M, Quarto F D, et al. The influence of nitrogen incorporation on the optical properties of anodic $\mathrm{Ta}_{2} \mathrm{O}_{5}$ Electrochim. Acta, 2012, 59: 382-386.

[5] Lu T, Wen J, Qian S, et al. Enhanced osteointegration on tantalum-implanted polyetheretherketone surface with bone-like elastic modulus. Biomater, 2015, 51:173-183.

[6] Long Z Y, Mitsuo N, Toshikazu A, et al. Corrosion resistance and biocompatibility of Ti-Ta alloys for biomedical applications. Mater. Sci. Eng. A, 2005, 398: 28-36.

[7] Geetha M, Singh A K, Asokamani R, et al. Ti based biomaterials, the ultimate choice for orthopaedic implants - A review. Prog Mater. Sci, 2009, 54: 397-425.

[8] Taheri M, Zahrani E M. Effect of novel thermal oxidation process on wear resistance of commercial pure titanium. Surf. Eng., 2008, 24(6): 475-478.

[9] Wiecinski P, Smolik J, Garbacz H, et al. Microstructure and mechanical properties of nanostructure multilayer $\mathrm{CrN} / \mathrm{Cr}$ coatings on titanium alloy. Thin Solid Films, 2011, 519(12): 4069-4073.

[10] Richard C, Kowandy C, Landoulsi J, et al. Corrosion and wear behavior of thermally sprayed nano ceramic coatings on commercially pure titanium and Ti- $13 \mathrm{Nb}-13 \mathrm{Zr}$ substrates. Int. J. $\mathrm{f}$ Refract. Met. H., 2010, 28(1):115-123.

[11] Fei C, Hai Z, Chen C, et al. Study on the tribological performance of ceramic coatings on titanium alloy surfaces obtained through microarc oxidation. Prog. Org. Coat., 2009, 64(2-3): 264-267.

[12] Tian Y S, Chen C Z, Li S T, et al. Research progress on laser surface modification of titanium alloys. Appl. Surf. Sci., 2005, 242(1-2): 177-184.

[13]A Zielinski, Jazdzewska M, Lubinski J, et al. Effects of laser remelting at cryogenic conditions on microstructure and wear resistance of the Ti6Al4V alloy applied in medicine. Solid State Phenom., 2012, 183: 215-224.

[14] Zhang J B, Fan D, Sun Y N, et al. Microstructure and hardness of the laser surface treated titanium. Key Eng. Mat., 2007, 353358: 1745-1748.

[15] Bao R L, Yu H J, Chen C Z, et al. Development of laser cladding wear-resistant coating on titanium alloys. Surf. Rev. and Lett., 2006, 13(5): 645-654.

[16] Garg S P, Krishnamurthy N, Awasthi A, et al. The O-Ta (oxygentantalum) system. J. Phase Equilibria, 1996, 17(1): 63-77.

[17] Ratke L, Alkemper J. Modelling of phase separation in liquids with a miscibility gap. Advances in Colloid and Interface Science, 1995, 58:151-170.

[18] John F. Partical Differential Equations. 4th ed. Wiley and Sons, NY, 1982

[19] Kurz W, Giovanola B, Trivedi R. Theory of microstructural development during rapid solidification. Acta Metall. Mater. , 1986, 34: 823-830.

[20] Eric A B. Smithells Metals Reference Book. Sixth ed. Robert Hartholl Ltd., 1983: 8-1.

[21] Lima M S F, Goldenstein H. Morphological instability of the austenite growth front in a laserremelted iron-carbon-silicon alloy. J. Cryst. Growth, 2000, 208: 709-716.

[22] Steen W M, Mazumder J. Laser Material Processing, 4th ed. Springer-Verlag London Limited: London, UK, 2010: 317-318. 